\title{
Uridine-cytidine kinase 2 promotes metastasis of hepatocellular carcinoma cells via the Stat3 pathway
}

This article was published in the following Dove Press journal: Cancer Management and Research

\author{
Qiming Zhou ${ }^{1,2, *}$ \\ Hai Jiang ${ }^{1-3, *}$ \\ Jianlong Zhang ${ }^{1,2, *}$ \\ Wei Yu ${ }^{1,2}$ \\ Zhenyu Zhou ${ }^{1,2}$ \\ Pinbo Huang ${ }^{1,2}$ \\ Jie Wang ${ }^{1,2}$ \\ Zhiyu Xiao ${ }^{1,2}$
}

'Guangdong Province Key Laboratory of Malignant Tumor Epigenetics and Gene Regulation, Sun Yat-Sen Memorial Hospital, Sun Yat-Sen University, Guangzhou 5I0I20, China; ${ }^{2}$ Department of Hepatobiliary Surgery, Sun Yat-Sen Memorial Hospital, Sun Yat-Sen University, Guangzhou 5 I0I20, China; ${ }^{3}$ Department of General Surgery, The First Affiliated Hospital of Nanchang University, Nanchang 330006, Jiangxi Province, China

*These authors contributed equally to this work

Correspondence: Jie Wang; Zhiyu Xiao Department of Hepatobiliary Surgery, SunYat-Sen Memorial Hospital, Sun Yat-Sen University, 107 Yanjiangxi Road, Guangzhou 5I0I20, China

Tel +86 I38 02900227

Fax +86 I36 82283695

Emailsumsjw@163.com;

xzysurgeon@163.com
Introduction: Uridine-cytidine kinase 2 (Uck2) has been demonstrated to activate antitumor prodrugs and regulate chemosensitivity in cancer cells. However, the expression and function of Uck2 in hepatocellular carcinoma (HCC) remain unknown. In this study, we were the first to systematically elucidate the role of Uck2 in HCC.

Patients and methods: Uck2 gene expression was queried between normal liver tissues and $\mathrm{HCC}$ in a web-based data mining platform (www.oncomine.org). Uck2 gene expression in tissue microarray was determined by immunohistochemical staining. The clinical and prognostic significance of Uck2 expression was statistically analyzed. Stable cell lines with increased Uck2 expression were established using lentivirus-based vectors, and RNAi technology was used to transiently downregulate Uck 2 expression. Cell migration and invasion were assessed by using wound-healing and transwell assays, respectively. mRNA and protein expression levels were determined using quantitative real-time PCR and Western blotting, respectively.

Results: We report the upregulation of Uck2 expression in HCC tissues. We explored the relationship between Uck2 levels and the clinicopathological features of HCC patients. High Uck2 notably correlated with early recurrence and poor prognosis in HCC patients. Uck2 expression in HCC cell lines regulated the cell migration and invasion capacities in vitro. The stable overexpression of Uck2 in Bel-7402 cells promoted their metastasis ability in vivo. Furthermore, the Uck2 upregulation increased the MMP2/9 expression and activated the Stat 3 signaling pathway. In addition, WP1066, a Stat3 inhibitor, neutralized the effects of Uck2 on the MMP2/9 expression and the migration and invasion capacities of HCC cells.

Conclusion: Our data suggest that Uck2 promotes HCC cell migration and invasion via the Stat3 signaling pathway and might be a novel potential target in HCC therapy.

Keywords: uridine-cytidine kinase 2, metastasis, hepatocellular carcinoma, oncogene, signal transducer, activator of transcription 3

\section{Introduction}

Hepatocellular carcinoma (HCC) is a common malignant tumor worldwide. ${ }^{1}$ In China, HCC accounts for one-fifth of the incidence of malignant tumors. ${ }^{2}$ Despite continuous progress in the treatment of HCC in the past decades, including advances in surgical resection, radiotherapy, chemotherapy, and liver transplantation, the high recurrence rate keeps the 5-year survival rate at less than $30 \% .{ }^{3}$ Studying the molecular features of HCC and identifying therapeutic targets for HCC are extremely urgent goals.

The Uck2 gene, located on chromosome 1q22-23.2, encodes uridine-cytidine kinase 2 (Uck2), which is involved in the salvage pathway of pyrimidine-nucleotide biosynthesis. ${ }^{4}$ Uck 2 generally catalyzes the conversion of uridine and cytidine to the 
monophosphate form using adenosine triphosphate (ATP) as the phosphate donor. ${ }^{5,6}$ Many studies have also indicated that Uck2 can catalyze the phosphorylation of nucleoside analogs and plays a crucial role in chemotherapy against cancer. Muhale et al demonstrated that knockdown of Uck2, which was involved in 5-fluorouridine $5^{\prime}$-monophosphate (FUMP) synthesis from 5-FU, produced a phenotype of cell sensitization to 5-FU.7 Murata et al suggested that Uck2 is responsible for the phosphorylation and activation of antitumor 3 '-ethynyl nucleosides. ${ }^{8}$ Sato et al indicated that Uck2 phosphorylates the nucleosidic medicine 3 '-ethynylcytidine (ECyd), resulting in increased drug sensitivity in cancer cells. ${ }^{9}$ The effects of Uck 2 on tumor progression have recently received increased attention. Shen et al suggested that Uck2 expression is elevated in breast cancer tissue and associated with poor overall survival. ${ }^{10}$ However, the potential prognostic value of Uck2 and its effect on the malignant biological behavior of cancer cells are not fully understood, particularly in HCC.

Signal transducer and activator of transcription 3 (Stat3) is a transcription factor encoded by the Stat 3 gene in humans. Persistent activation of Stat 3 has been frequently observed in many types of tumors, ${ }^{11}$ including HCC. ${ }^{12,13}$ The activation of Stat3 modulates the expression of genes that promote tumor cell survival, proliferation, metastasis, and angiogenesis. ${ }^{11,14}$ Thus, the targeted inhibition of the Stat 3 signaling pathway may provide significant therapeutic benefits for $\mathrm{HCC}$ patients.

Here, we are the first to demonstrate the important role of Uck2 in HCC. We explored the expression levels of Uck2 in HCC tissues and normal controls. We further investigated the relationship between Uck2 expression and both clinicopathological features and prognosis in HCC patients. In vitro and in vivo models were used to evaluate the effects of Uck2 on the migration, invasion, proliferation, and apoptosis of HCC cells. Finally, we partially elucidated the potential mechanism by which Uck 2 regulates migration and invasion in HCC cells. Taken together, we provided the basis for the potential use of Uck2 as a novel therapeutic target for HCC in the future.

\section{Materials and methods}

\section{Cell lines and reagents}

HEK293T cells and the HCC cell lines LO2, HepG2, Skhep-1, PLC/PRF/5, SMMC-7721, Bel-7402, MHCC97H, and HCCLM6 were purchased from the Cell Bank of the Chinese Academy of Sciences (Shanghai, China). The cell lines were maintained in either DMEM or Roswell Park Memorial Institute (RPMI)-1640 medium supplemented with 10\% FBS (all from Gibco, Thermo Fisher Scientific, Waltham, MA, USA) in an atmosphere of $5 \% \mathrm{CO}_{2}$ at $37^{\circ} \mathrm{C}$. WP1066, an inhibitor of Stat3, was purchased from Medchem Express (Monmouth Junction, NJ, USA)

\section{Oncomine database analysis}

Oncomine is a web-based data mining platform (www. oncomine.org). The gene search function of Oncomine was used to locate microarray studies for which gene expression data were publicly available. Uck2 gene expression was queried between normal liver tissues and HCC. Data from microarray studies were processed by Oncomine, and the results obtained were presented in box plots, along with the gene rank, fold change, and statistical analysis. Query results from two studies showed significant increases in Uck2 gene expression in HCC.

\section{Patients and tissue specimens}

Paraffin-embedded tumors and adjacent liver tissue specimens (at least $2 \mathrm{~cm}$ away from the tumor edge) for tissue microarray (TMA) were collected from a series of 90 patients with HCC who had undergone hepatic resection between January 1, 2012, and December 31, 2012, at Sun Yat-sen Memorial Hospital, Sun Yat-sen University (SYSU), Guangzhou, China. None of the patients underwent preoperative chemotherapy or interventional radiology. The study protocol was approved by the Institute Review Board of SYSU, and informed consent was obtained from each patient. The procedure was conducted in accordance with the Declaration of Helsinki. The tissue samples of these patients were frozen immediately in liquid nitrogen and stored at $-80^{\circ} \mathrm{C}$ for RNA extraction. Approximately $5-\mathrm{mm}^{2} \mathrm{HCC}$ tissue samples that contained $>80 \%$ tumor cells and lacked necrotic tissue were used for RNA extraction. Detailed patient information is listed in Table 1. The diagnosis and pathological grades of HCC patients were reviewed separately by two histopathologists. The presence or absence of complete tumor encapsulation of HCC focus was independently judged by two radiologists after reviewing all available magnetic resonance imaging sequences. The presence of complete encapsulation was based on the following criteria: a clearly defined enhanced, high-signal-intensity rim on postcontrast portal or delayed-phase T1-weighted images and/or a low-signal-intensity rim on T1-weighted and T2-weighted images encompassing the entire HCC focus circumference. Microvascular invasion was defined as the presence of a thrombus formed by tumor cells in a vascular space lined by endothelial cells, which was located in the tumor parenchyma or in the surrounding liver parenchyma. 
Table I Correlation of Uck2 expression with patient's clinicopathologic features in HCCs

\begin{tabular}{|c|c|c|c|c|}
\hline \multirow[t]{2}{*}{ Clinicopathological features } & \multirow[t]{2}{*}{$\mathbf{n}$} & \multicolumn{2}{|c|}{ Uck2 expression } & \multirow[t]{2}{*}{$P$-value ${ }^{a}$} \\
\hline & & $\begin{array}{l}\text { Low } \\
\text { expression } \\
(n=42)\end{array}$ & $\begin{array}{l}\text { High } \\
\text { expression } \\
(n=48)\end{array}$ & \\
\hline Age & & $51.9 \pm 12.4$ & $53.2 \pm 13.2$ & 0.806 \\
\hline \multicolumn{5}{|l|}{ Gender } \\
\hline Female & 48 & $27(64.3 \%)$ & 21 (43.8\%) & 0.082 \\
\hline Male & 42 & 15 (35.7\%) & $27(56.2 \%)$ & \\
\hline \multicolumn{5}{|l|}{ Serum AFP (ng/mL) } \\
\hline$\leq 20$ & 26 & $9(21.4 \%)$ & $17(35.4 \%)$ & 0.220 \\
\hline$>20$ & 64 & $33(78.6 \%)$ & 31 (64.6\%) & \\
\hline \multicolumn{5}{|l|}{ HBV infection } \\
\hline Negative & 24 & $8(19.0 \%)$ & $16(33.3 \%)$ & 0.197 \\
\hline Positive & 66 & $34(81.0 \%)$ & $32(66.7 \%)$ & \\
\hline \multicolumn{5}{|l|}{ Tumor number } \\
\hline Single & 79 & 36 (85.7\%) & $43(89.6 \%)$ & 0.813 \\
\hline Multiple & II & $6(14.4 \%)$ & $5(10.4 \%)$ & \\
\hline \multicolumn{5}{|l|}{ Tumor size $(\mathrm{cm})$} \\
\hline$\leq 5$ & 20 & $8(19 \%)$ & $12(25 \%)$ & 0.672 \\
\hline$>5$ & 70 & $34(81 \%)$ & $36(75 \%)$ & \\
\hline \multicolumn{5}{|l|}{ Tumor encapsulation } \\
\hline Absent & 35 & $7(16.7 \%)$ & $28(58.3 \%)$ & $<0.00 I^{* *}$ \\
\hline Present & 55 & $35(83.3 \%)$ & $20(41.7 \%)$ & \\
\hline \multicolumn{5}{|l|}{ Microvascular invasion } \\
\hline Absent & 58 & $36(85.7 \%)$ & $22(45.8 \%)$ & $<0.00 I^{* *}$ \\
\hline Present & 32 & $6(14.3 \%)$ & $26(54.2 \%)$ & \\
\hline \multicolumn{5}{|l|}{ Edmondson-Steiner grade } \\
\hline $\mathrm{I} / \mathrm{II}$ & 55 & 25 (59.5\%) & $30(62.5 \%)$ & 0.942 \\
\hline III/IV & 35 & $17(40.5 \%)$ & $18(37.5 \%)$ & \\
\hline \multicolumn{5}{|l|}{ Metastasis } \\
\hline Absent & 43 & 29 (69.0\%) & I 4 (29.2\%) & $<0.001 * *$ \\
\hline Present & 47 & $13(31.0 \%)$ & 34 (70.8\%) & \\
\hline \multicolumn{5}{|l|}{ TNM stage } \\
\hline $\mathrm{I} / \mathrm{II}$ & 24 & II (26.2\%) & $13(27.7 \%)$ & 0.876 \\
\hline III/IV & 65 & 31 (73.8\%) & 34 (72.3\%) & \\
\hline
\end{tabular}

Note: aChi-squared test. $* * p<0.01$

Abbreviations: AFP, $\alpha$-fetoprotein; HBV, hepatitis B virus; TNM, tumor node metastasis; $n$, number of cases.

Metastasis was defined as the presence of intrahepatic satellite lesions of HCC or extrahepatic metastases in distant organs confirmed by imaging examinations in combination with histopathological diagnosis. The specimens were classified according to the American Joint Committee on Cancer (AJCC)-TNM Classification of Malignant Tumors.

\section{Quantitative real-time PCR (RT-qPCR)}

Total RNA was extracted using the RNAiso Plus reagent (Takara Bio, Shiga, Japan) and reverse transcribed into cDNA using a PrimeScript ${ }^{\mathrm{TM}}$ RT reagent kit (Takara Bio) according to the manufacturer's protocols. We performed RT-qPCR using $2 \times$ SYBR Green qPCR master mix (Bimake, Houston, TX, USA) in a $10-\mu \mathrm{L}$ volume reaction system.
The RT-qPCR conditions consisted of 45 cycles, with 15 seconds of denaturation at $95^{\circ} \mathrm{C}$ and 45 seconds of annealing at $60^{\circ} \mathrm{C}$. The following primers were used: Uck2, forward: 5'-CGGCGAGCCCTTCCTTATAG-3' and reverse: 5'- ATTGTCAAAGGCATCCGGGT-3'; GAPDH, forward: 5'-GAAGGTGAAGGTCGGAGTCAACG-3' and reverse: 5'-TGCCATGGGTGGAATCATATTGG-3'. The reactions were performed in triplicate. The relative expression level of the Uck2 gene was calculated based on the $2^{-} \Delta \Delta^{\mathrm{Cq}}$ method in cell lines and the $2^{-} \Delta^{\mathrm{Ct}}$ and $-\Delta \Delta \mathrm{Ct}$ methods in clinical samples.

\section{Protein extraction and Western blotting}

Cell lysates were harvested using radioimmunoprecipitation assay (RIPA) buffer (CWbio, Beijing, China) for 30 
minutes on ice and centrifuged at $13,000 \times \mathrm{g}$ for 25 minutes at $4{ }^{\circ} \mathrm{C}$. The protein concentration of the supernatant was determined by BCA assays (CWbio). Equal amounts of protein were separated by $6 \%, 10 \%$, and $12 \%$ SDS-PAGE and electrotransferred onto $0.22-\mu \mathrm{m}$ polyvinylidene difluoride membranes (EMD Millipore, Billerica, MA, USA). After stripping, the membrane was blocked in 5\% fat-free milk and incubated with primary antibodies overnight at $4{ }^{\circ} \mathrm{C}$. Then, the bands were washed with $0.1 \%$ Tris-buffered saline with Tween-20 (TBST) three times and incubated with horseradish peroxidase-conjugated secondary antibodies at room temperature for 2 hours. The protein signal on the bands was detected by using an enhanced chemiluminescence system (EMD Millipore) after washing with $0.1 \%$ TBST three times. The following anti-Homo sapiens primary antibodies were used for Western blotting: mouse anti- $\beta$-actin (1:5,000 dilution, Proteintech, 60008-1-Ig); rabbit anti-Uck2 (1:1,000 dilution, Proteintech, 10511-1AP); rabbit anti-MMP2 (1:1,000 dilution, ImmunoWay, YT2798); rabbit anti-MMP7 (1:1,000 dilution, ImmunoWay, YT2663); rabbit anti-MMP9 (1:1,000 dilution, ImmunoWay, YT1892); rabbit anti-E-cadherin (1:500 dilution, Abcam, ab15148); rabbit anti-N-cadherin (1:1,000 dilution, Abcam, ab76057); mouse anti-Viomentin (1:1,000 dilution, Abcam, ab8978); rabbit anti-fibronectin (1:1,000 dilution, Abcam, ab32419); rabbit anti-p44/42 MAPK (Erk1/2) (1:2,000 dilution, CST, \#4695); rabbit anti-phospho-p44/42 MAPK (Erk1/2) (Thr202/Tyr204) (1:2,000 dilution, CST, \#4370); mouse anti-Stat3 (1:2,000 dilution, CST, \#9139); rabbit anti-phospho-Stat3 (Try705) (1:2,000 dilution, CST, \#9145); mouse anti-Akt (1:2,000 dilution, CST, \#2920); and rabbit anti-phospho-Akt (Ser473) (1:2,000 dilution, CST, \#4060). The following secondary antibodies were used for Western blotting: goat anti-mouse (1:10,000 dilution, sc-2005; Santa Cruz Biotechnology, Inc., Dallas, TX, USA) and goat anti-rabbit (1:10,000 dilution, sc-2004; Santa Cruz Biotechnology, Inc.).

\section{TMA and immunohistochemistry}

Immunohistochemical (IHC) staining was performed by using TMA block sections to detect Uck2 (1:100 dilution, Abcam, ab60222) expression in patient samples. The HCC tumor tissues and adjacent liver tissues were re-embedded from each pair of blocks to recipient blocks. For immunohistochemistry, 4- $\mu \mathrm{m}$ sections from the TMA blocks were obtained using a microtome. IHC staining was performed using a Dako Envision Plus System (Dako, Carpinteria, CA, USA) according to the manufacturer's protocol. The immunostained sections were evaluated independently by two pathologists who were blinded to the clinicopathological data. The staining intensity was scored as follows: 0 (negative); 1 (weak); 2 (moderate); and 3 (strong). The proportion of positive-stained cells was scored as follows: $0, \leq 10 \% ; 1,11 \%-25 \% ; 2,26 \%-50 \% ; 3,51 \%-75 \%$; and 4, 76\%-100\%. IHC staining total scores were obtained by multiplying the intensity score by the proportion score; cases with a total score of $0-3$ were defined as low expression, and cases with a total score of 4-12 were defined as high expression.

\section{Knockdown of Uck2 expression using Uck2-specific siRNAs}

Small interfering RNAs (siRNAs) specific for Uck2 (siRNA\#1: 5'-GCGGCGAGCCCUUCCUUAUTT-3' and siRNA-\#2: 5'-GCCAGAAGCAGGUGGUCAUTT-3') (GenePharma, Shanghai, China) were used to transfect Sk-hep-1 and HCCLM6 cells, which express relatively high levels of Uck2. The negative control siRNA served as a control nontargeting siRNA (N.C.). Sk-hep-1 and HCCLM6 cells were seeded into 6-cm dishes containing $5 \mathrm{~mL}$ of DMEM with $10 \%$ FBS. After the density of the cells reached nearly $75 \%$ within 24 hours, the cells were transfected with 600 pmol of siRNA-N.C. or siRNA-\#1 and siRNA-\#2 in the presence of $10 \mu \mathrm{L}$ of Lipofectamine ${ }^{\circledR} 3000$ (Invitrogen, Thermo Fisher Scientific, Waltham, MA, USA). After 48 hours of incubation following siRNA transfection, total RNA and protein were extracted, and the cells were used for further functional assays after treatment with $0.25 \%$ trypsin-EDTA (Gibco, Thermo Fisher Scientific).

\section{Establishing stable cell lines with increased Uck2 expression}

The Uck2 open reading frame (GenBank: NM-012474.4) was cloned into the lentiviral vector pCDH (GENEray, Shanghai, China). A lentivirus delivering $\mathrm{pCDH}$-control or pCDH-Uck2 was generated from $293 \mathrm{~T}$ cells and was used to infect SMMC7721 cells and Bel-7402 cells. After 72 hours of infection, selective culture medium containing $2 \mu \mathrm{g} / \mathrm{mL}$ puromycin was used to select cells with stable expression of Uck 2 for 2 weeks. Stable expression of Uck2 in HCC cells was detected by RT-qPCR and Western blot analysis.

\section{Analysis of the cell cycle by flow cytometry}

For each group, $2 \times 10^{6}$ cells were harvested, washed twice, and fixed with $500 \mu \mathrm{L}$ of ice-cold $70 \%$ ethanol at $4{ }^{\circ} \mathrm{C}$ over- 
night. The cells were then washed with PBS containing ribonuclease and stained with $800 \mu \mathrm{L}$ of propidium iodide (PI; $50 \mu \mathrm{g} / \mathrm{mL}$ ) for 30 minutes at room temperature. The stained cells were analyzed with a flow cytometer using Beckman Coulter (Brea, CA, USA) and ModFit (Verity, Topsham, ME, USA) software.

\section{Analysis of apoptosis by flow cytometry}

An Annexin V/PI apoptosis detection kit I (BD Biosciences, San Jose, CA, USA) was used to measure apoptosis. The cells were washed twice and resuspended at a density of $1 \times 10^{6}$ cells $/ 200 \mu \mathrm{L}$ in binding buffer with $10 \mu \mathrm{L}$ of PI and $10 \mu \mathrm{L}$ of Annexin V-FITC. After incubation at room temperature for 5 minutes in the dark, the cells were subjected to flow cytometry for apoptosis analysis.

\section{MTT proliferation assay}

An MTT assay was used to evaluate cell proliferation according to the manufacturer's protocol. Briefly, cells (1,000 cells in $100 \mu \mathrm{L} /$ well) were seeded in triplicate into a 96-well plate and incubated for an additional 24-72 hours. After incubation for a specific period of time, $20 \mu \mathrm{L}$ of MTT solution ( $5 \mathrm{mg}$ / $\mathrm{mL}$; Sigma-Aldrich Co., St Louis, MO, USA) was added to each well and incubated with the cells for 4 hours. Then, $150 \mu \mathrm{L}$ of dimethyl sulphoxide (DMSO; Sigma-Aldrich Co.) was added to each well, and the cells were incubated for another 15 minutes. The absorbance was read at $570 \mathrm{~nm}$ using a microplate reader.

\section{In vitro migration and invasion assays}

Migration and invasion assays were performed using uncoated or Matrigel-coated (BD Biosciences) transwell culture chambers $(8.0-\mu \mathrm{m}$ pore size; Corning Inc., Corning, NY, USA) according to the manufacturer's instructions. First, the transwell culture chambers were placed onto 24-well plates. For migration assays, the cells were resuspended in serumfree medium $\left(8 \times 10^{5}\right.$ cells $/ \mathrm{mL}$ for Bel- 7402 cells, $4 \times 10^{5}$ for Sk-hep-1 cells), and $100 \mu \mathrm{L}$ of the suspension was added to the upper chambers. For invasion assays, the cells were resuspended in serum-free medium $\left(10 \times 10^{5}\right.$ for Bel-7402 cells, $8 \times 10^{5}$ for Sk-hep- 1 cells), and $100 \mu \mathrm{L}$ of the suspension was added into the upper chambers, which were coated with $50 \mu \mathrm{L}$ $(1.25 \mathrm{mg} / \mathrm{mL})$ of BD Matrigel ${ }^{\mathrm{TM}}$ Matrix (BD Biosciences). The lower chambers were both filled with $400 \mu \mathrm{L}$ of RPMI1640 or DMEM containing 15\% FBS. Following a 24-hour incubation for the migration assay and a 48-hour incubation for the invasion assay, the cells on the lower surface of the upper chambers were fixed in $4 \%$ paraformaldehyde, stained with $0.25 \%$ crystal violet for 20 minutes, and counted in five random fields at a magnification of $100 \times$. Each experiment was independently repeated in triplicate.

\section{Wound-healing assay}

$\mathrm{HCC}$ cells were seeded in 6-well plates and grown to $90 \%$ confluence. A wound in the cell monolayer was created by gently scraping with a sterile pipette tip. Floating cells were removed with $\mathrm{PBS}$, and the cultures were maintained in DMEM supplemented with $2 \%$ FBS. The cells were observed, and images were captured at 0 and 24 hours after scraping.

\section{In vivo metastasis}

Female BALB/c nude mice aged 4-6 weeks were purchased from the Laboratory Animal Center of SYSU and maintained in sterile laminar flow cabinets. The cells (Bel-7402-control and Bel-7402-Uck2) were resuspended in PBS at a concentration of $2 \times 10^{7}$ cells $/ \mathrm{mL}$. The cell suspension $(0.1 \mathrm{~mL})$ was injected into the tail veins of nude mice. The mice were sacrificed 8 weeks after injection. The lungs were collected, fixed in 4\% paraformaldehyde and embedded in paraffin, followed by serial sectioning. Five sections (100 $\mu \mathrm{m}$ apart) from each lung were stained with H\&E. All experimental procedures involving animals were in accordance with the National Institutes of Health guide for the care and use of laboratory animals and approved by the Animal Ethical and Welfare Committee of SYSU.

\section{Statistical analysis}

All statistical analyses were performed with SPSS 23.0 or GraphPad Prism 6.0. Student's $t$-test was used to compare Uck2 mRNA levels and the number of metastatic lung foci in paired samples and densitometry analysis of Western blotting. The Mann-Whitney test was used to compare the proportions of the staining intensity scores from IHC in the high- and low-Uck2 expression groups. The chi-squared test was used to analyze the relationship between Uck2 expression and clinicopathological features. Kaplan-Meier plots and log-rank tests were used to assess overall survival and recurrence-free survival. Univariate and multivariate analyses were performed by the log-rank test and the Cox proportional hazards regression model, respectively. $P$ values $<0.05$ were considered to be statistically significant.

\section{Results \\ Uck2 is overexpressed in HCC}

To determine whether Uck2 gene expression is associated with $\mathrm{HCC}$, we first carried out an Oncomine ${ }^{\mathrm{TM}}$-based database query. ${ }^{15}$ The analysis of the data from two previous 
reports is shown in Figure 1. Chen et al were the first to demonstrate $>2$-fold-higher Uck2 expression in tumors compared with non-tumor liver tissue $(P<0.0001)$ (Figure 1A). ${ }^{16}$ By comparing a large number of samples, Roessler et al also showed that Uck2 mRNA levels were 2.721-fold higher in tumor cores than in normal liver tissues and that Uck2 ranked amongst the top 1\% of overexpressed genes in tumor cells ( $t=24.015, P<0.0001)$ (Figure 1B). ${ }^{17}$ These lines of evidence suggested that Uck2 gene expression might be associated with HCC. Therefore, we investigated the data of patients in our hospital. We evaluated the expression of Uck 2 mRNA in 90 pairs of HCC and non-tumor tissues by RT-qPCR. As shown in Figure $1 C$ and D, we also found higher expression levels of Uck 2 mRNA in these HCC tissues than in non-tumor tissues. Together, these data clearly indicate that the Uck2 gene is overexpressed in HCC.

\section{High Uck2 expression correlates with aggressive clinicopathological features and poor prognosis in $\mathrm{HCC}$}

IHC was used in a TMA that included 90 pairs of HCC and adjacent liver tissues to further investigate the protein expression of Uck2 in HCC. The results showed that Uck2 was mainly localized in the cytoplasm of hepatocytes and HCC cells (Figure 2A and B). There was no difference in the cellular localization of Uck 2 between HCC tissues and adjacent liver tissues. Forty-eight of the 90 (53.3\%) paraffinembedded HCC tissues showed high Uck2 expression, while
A

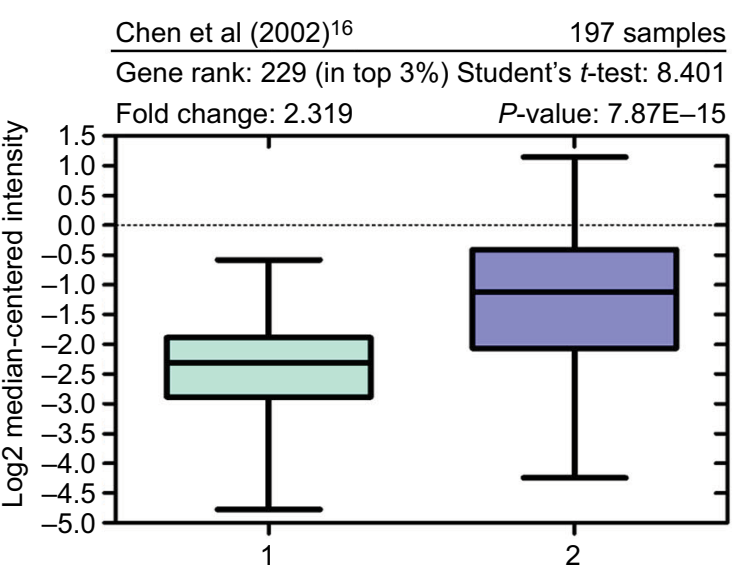

C

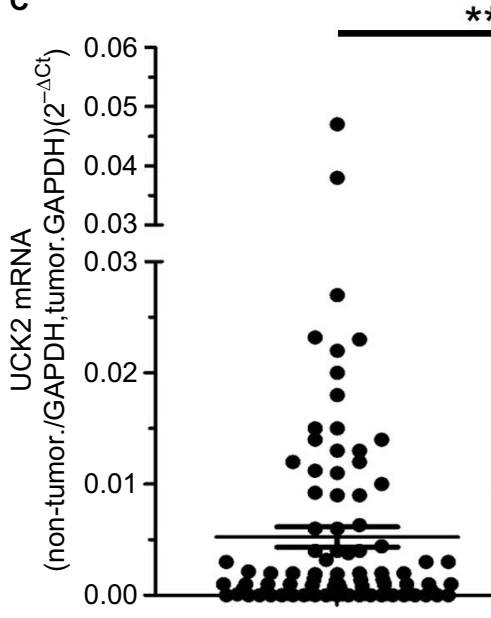

non-tumor
**

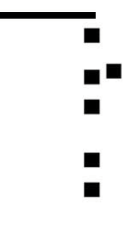

B

Roessler et al (2010) ${ }^{17}$

445 samples

Gene rank: 87 (in top 1\%) Student's $t$-test: 24.015

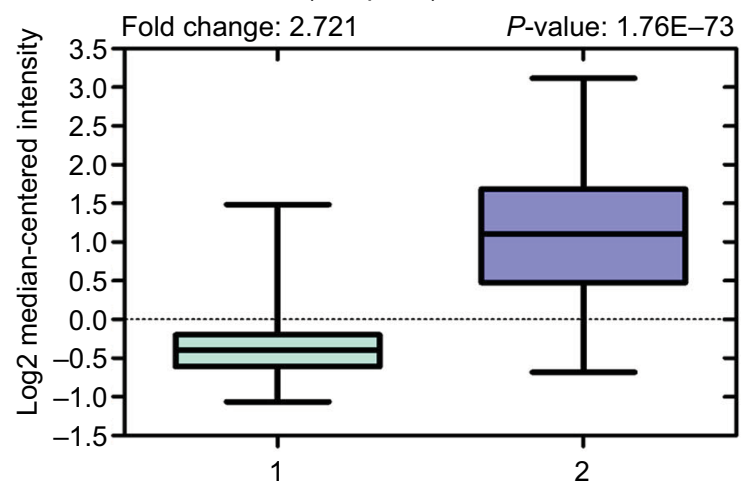

D

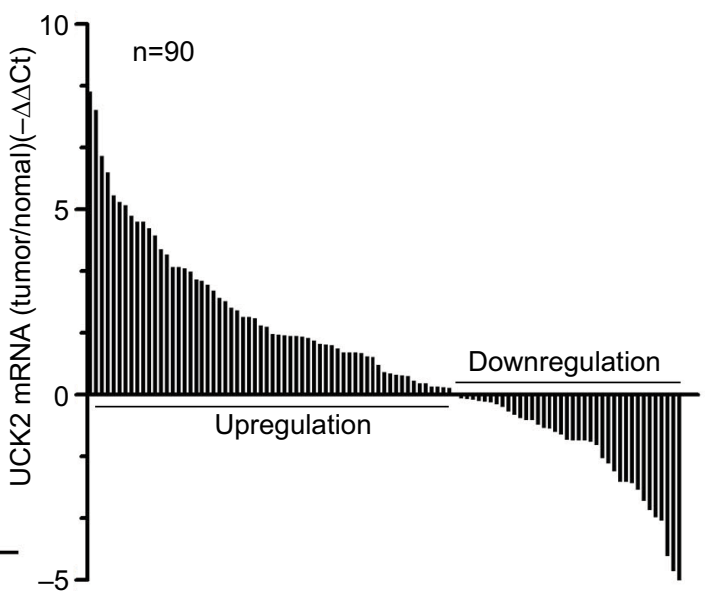

Figure I Uck2 gene is overexpressed in hepatocellular carcinoma.

Notes: Oncomine-based microarray database query of Uck2 gene expression was performed. Two different reports were queried: (A) Chen et al ${ }^{16}$ and (B) Roessler et al. ${ }^{17}$ The studies compared Uck2 mRNA levels in non-tumor liver tissues and tumor cores. The overexpression gene rank for Uck2, the number of samples, statistical analysis, fold changes, and $P$-values were provided at the top of the box-and-whisker plots, which were obtained in the Oncomine query. (C) and (D) Elevated expression of Uck2 mRNA in 90 pairs of $\mathrm{HCC}$ tissues and adjacent non-tumor liver tissues was detected by RT-qPCR. Data are shown as the $2^{-} \Delta^{\mathrm{Ct}}$ and $-\Delta \Delta C \mathrm{Ct}$ values $(* * P<0.0 \mathrm{I})$.

Abbreviation: Uck2, uridine-cytidine kinase 2. 

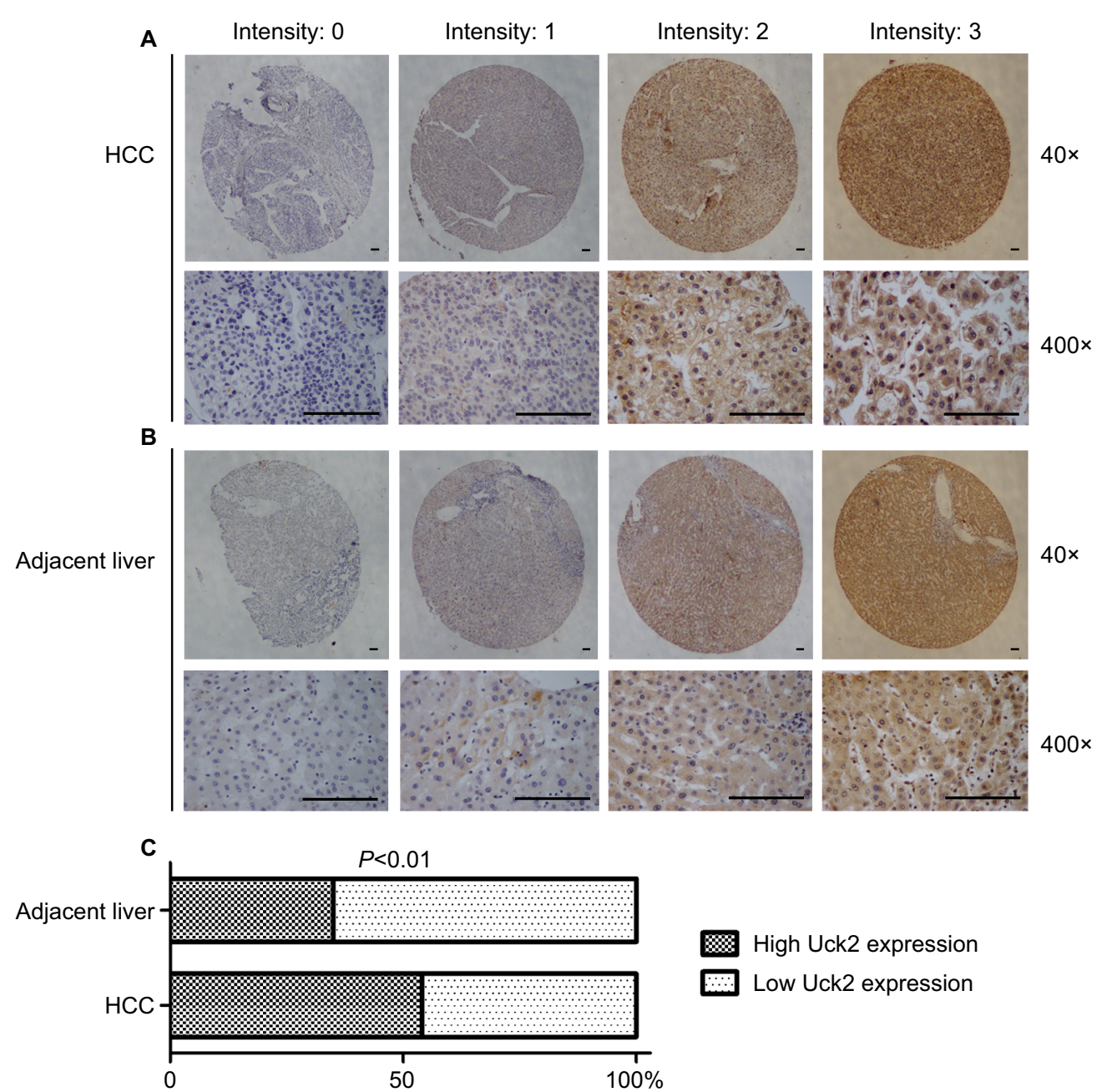

D

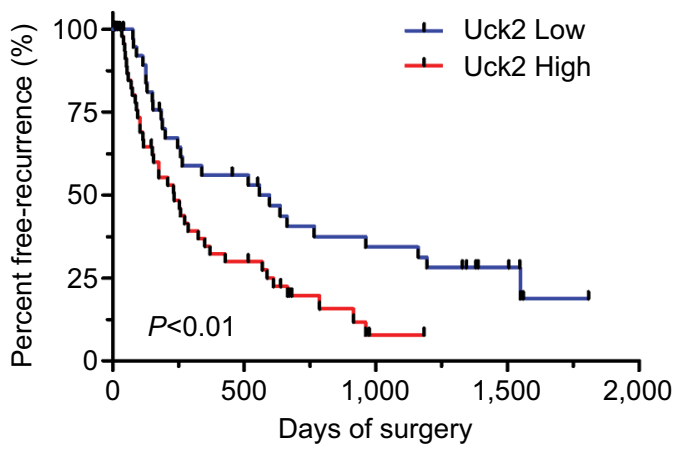

E

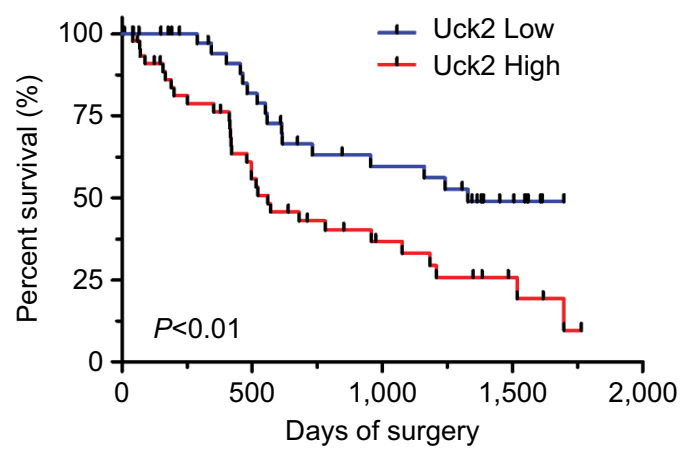

Figure 2 High Uck2 expression correlates with aggressive clinicopathological features and poor prognosis in HCC.

Notes: (A) and (B) Immunohistochemical analysis of Uck2 expression in a TMA of 90 pairs of HCC and adjacent non-tumor tissues (scale bar, I00 $\mu$ m). The staining intensity was scored as follows: 0 (negative), I (weak), 2 (moderate), and 3 (strong). (C) The component ratios of IHC staining between HCC tissues and adjacent liver tissues were obviously different, as analyzed by the Mann-Whitney test $(P<0.0 \mathrm{I})$. (D) and (E) Kaplan-Meier analysis of recurrence-free survival and overall survival for HCC patients based on the TMA. HCC patients with high Uck2 expression had shorter recurrence-free survival and overall survival than patients with low Uck2 expression. IHC staining total scores were obtained through multiplying the intensity score by the proportion score; total scores of 4-12 were defined as high Uck2 expression, and total scores of 0-3 were defined as low Uck2 expression. $P<0.05$ indicates a significant difference.

Abbreviations: HCC, hepatocellular carcinoma; IHC, immunohistochemical; TMA, tissue microarray; Uck2, uridine-cytidine kinase 2.

42 of the 90 (46.7\%) HCC tissues showed low Uck2 expression. Thirty of the 90 (33.3\%) adjacent liver tissues exhibited high Uck2 expression, and the remaining 60 (66.7\%) adjacent liver tissues showed low Uck2 expression. The component ratios of the high and low Uck2 expression between HCC tissues and adjacent liver tissues were obviously different $(z=-2.70, P=0.007)$ (Figure 2C). We next examined the correlations between Uck2 expression and clinicopathological 
features, recurrence-free survival, and overall survival in HCC patients, as indicated by TMA. High Uck2 expression was found to significantly correlate with tumor encapsulation, microvascular invasion, and distant metastasis (all $P$-values $<0.001$, Table 1). Kaplan-Meier survival analysis indicated that a higher expression level of Uck2 was significantly associated with shorter recurrence-free survival and overall survival (Figure 2D and E). The median recurrence-free survival in HCC patients with low Uck2 expression was 557 days, compared with 234 days in patients with high Uck2 expression (Figure 2D, $P$-value=0.007). The 5 -year overall survival rate of the patients with low Uck2 expression was $41.6 \%$, while that of the patients with Uck2 high expression was $19.4 \%$ (Figure $2 \mathrm{E}, P$-value=0.004). In addition to the Uck2 expression level, the integrity of tumor encapsulation, microvascular invasion, distant metastasis, and TNM stage significantly correlated with overall survival in the univariate analysis (Table 2). The Cox proportional hazards model was further employed to evaluate the effects of independent factors on overall survival. These factors included Uck2 expression, tumor number, tumor size, the integrity of tumor encapsulation, microvascular invasion, tumor differentiation, and TNM stage. The results showed that Uck2 expression, tumor number, and microvascular invasion were independent prognostic factors for survival (Table 2). Therefore, multivariate analysis indicated that Uck 2 protein expression has a significant correlation with poor prognosis in HCC patients as an independent factor $(\mathrm{HR}=0.369,95 \% \mathrm{CI}=0.166-0.820$, $P$-value $=0.014)$.

\section{Uck2 promoted HCC cell migration and invasion in vitro}

Western blotting was performed to determine Uck2 protein levels in a normal liver cell line and HCC cell lines (Figure 3A1). To evaluate the role of Uck2 in HCC, SMMC-7721 (low Uck2 expression), Bel-7402 (low Uck2 expression), Sk-hep-1 (high Uck2 expression), and HCCLM6 (high Uck2 expression) cells were investigated further. We infected Bel-7402 cells and SMMC7721 cells with lentiviruses that ectopically express Uck2. The level of Uck2 overexpression in Bel-7402 cells was confirmed by RT-qPCR and Western blotting, with a 6-fold upregulation in mRNA level and a nearly 2.5-fold upregulation in protein content (Figure 3B). We then employed wound-healing and transwell assays to detect the effect of Uck2 gene on $\mathrm{HCC}$ cell migration and invasion. As shown in Figure 3D1, an evident acceleration

Table 2 Univariate and multivariate analysis of prognostic factors of overall survival in Uck2 patients

\begin{tabular}{|c|c|c|c|c|c|c|}
\hline \multirow[t]{2}{*}{ Characteristic } & \multicolumn{2}{|c|}{ Univariate analysis } & \multirow[b]{2}{*}{$P$-value } & \multicolumn{2}{|c|}{ Multivariate analysis } & \multirow[b]{2}{*}{$P$-value } \\
\hline & HR & $95 \% \mathrm{Cl}$ & & HR & $95 \% \mathrm{Cl}$ & \\
\hline \multicolumn{7}{|l|}{ Uck2 expression } \\
\hline Low vs high & $0.4 \mid 4$ & $0.222-0.773$ & $0.006 * *$ & 0.369 & $0.166-0.820$ & $0.014 *$ \\
\hline \multicolumn{7}{|l|}{ Age (years) } \\
\hline$<50$ vs $\geq 50$ & 1.011 & $0.986-1.036$ & 0.399 & & & \\
\hline \multicolumn{7}{|l|}{ Gender } \\
\hline Female vs male & 1.642 & $0.897-3.006$ & 0.108 & & & \\
\hline \multicolumn{7}{|l|}{ Serum AFP (ng/mL) } \\
\hline$\leq 20$ vs $>20$ & 1.463 & $0.790-2.712$ & 0.226 & & & \\
\hline \multicolumn{7}{|l|}{ HBV infection } \\
\hline Negative vs positive & 1.067 & $0.522-2.183$ & 0.859 & & & \\
\hline \multicolumn{7}{|l|}{ Tumor number } \\
\hline Single vs multiple & 2.563 & I. $176-5.588$ & $0.018 *$ & 0.202 & $0.077-0.530$ & $0.001 *$ \\
\hline \multicolumn{7}{|l|}{ Tumor size $(\mathrm{cm})$} \\
\hline$\leq 5$ vs $>5$ & 0.757 & $0.382-1.50 \mathrm{I}$ & 0.425 & 0.651 & $0.292-1.45 \mathrm{I}$ & 0.294 \\
\hline \multicolumn{7}{|l|}{ Tumor encapsulation } \\
\hline Absent vs present & 1.932 & $1.064-3.509$ & $0.03 I^{*}$ & 1.337 & $0.572-3.125$ & 0.503 \\
\hline \multicolumn{7}{|l|}{ Microvascular invasion } \\
\hline Absent vs present & 0.396 & $0.219-0.716$ & $0.002 * *$ & 0.392 & $0.165-0.930$ & $0.034^{*}$ \\
\hline \multicolumn{7}{|l|}{ Edmondson-Steiner grade } \\
\hline I-II vs III/IV & 0.982 & $0.536-1.802$ & 0.954 & 0.655 & $0.324-1.325$ & 0.239 \\
\hline \multicolumn{7}{|l|}{ Metastasis } \\
\hline Absent vs present & 0.502 & $0.274-0.920$ & $0.026^{*}$ & 0.848 & $0.394-1.826$ & 0.673 \\
\hline \multicolumn{7}{|l|}{ TNM stage } \\
\hline I/II vs III/IV & 0.489 & $0.24 I-0.99 \mid$ & $0.047^{*}$ & 0.753 & $0.324-1.753$ & $0.5 \mathrm{II}$ \\
\hline
\end{tabular}

Note: $* P<0.05, * * P<0.01$.

Abbreviations: AFP, $\alpha$-fetoprotein; HBV, hepatitis B virus; TNM, tumor node metastasis; HR, hazard ratio. 
A1

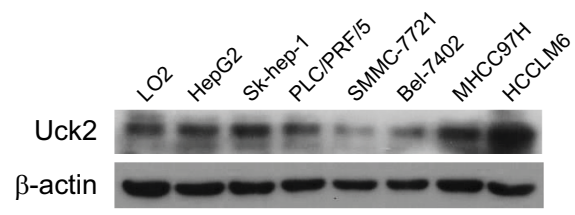

B
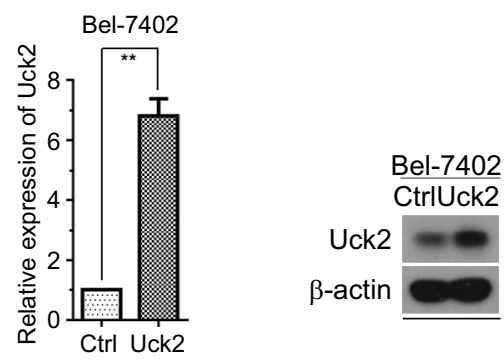

A2

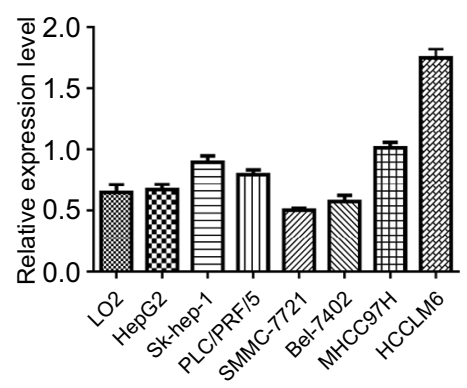

C

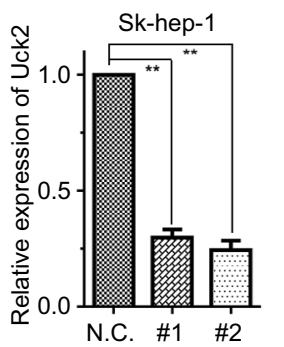

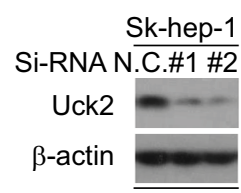

D1

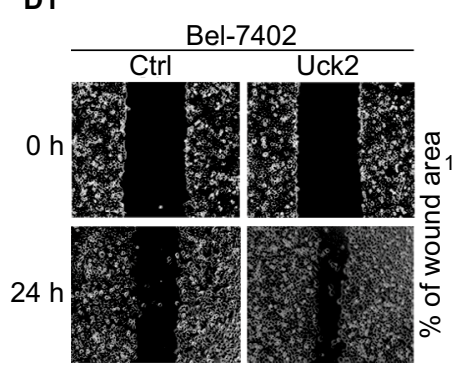

D2
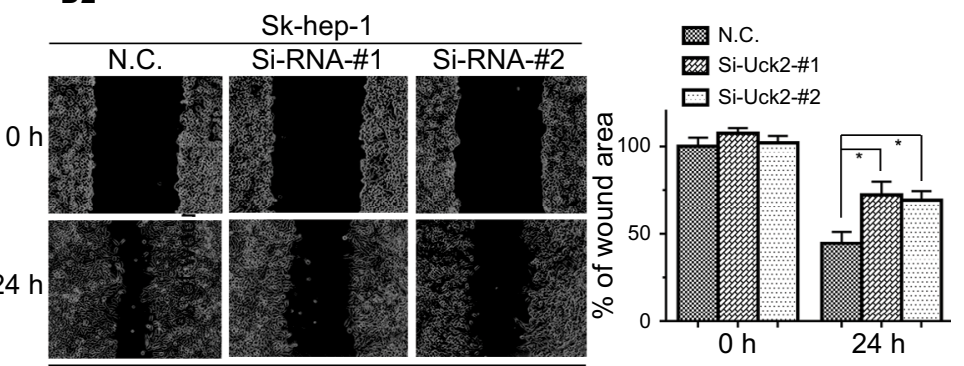

Figure 3 Uck2 promoted HCC cell migration and invasion in vitro.

Notes: (Al and A2) Raw photos and densitometry analysis of Western blotting: endogenous Uck2 expression in one normal liver cell line and seven human HCC cell lines. $\beta$-actin was used as the housekeeping gene to normalize expression levels in the densitometry analysis by Image software(I.48 version; NIH, Bethesda, MD, USA). (B) Stable Uck2 overexpression in Bel-7402 cells infected with the ectopic expression vector, as detected by RT-qPCR and Western blotting. (C) A specific Uck2 siRNA resulted in the reduction of Uck2 expression in Sk-hep-I cells, as detected by RT-qPCR and Western blotting. (DI and D2) Effects of Uck2 overexpression and knockdown on HCC cell migration by a wound-healing assay. The experiments were repeated three times, and the results are shown as the mean $\pm S D$; $* P<0.05$; $* * P<0.01$, which indicates a significant difference by an independent Student's $t$-test.

Abbreviations: Ctrl, control; CV, coefficient of variation; HCC, hepatocellular carcinoma; N.C., negative control; Si, Si-RNA; Uck2, uridine-cytidine kinase 2.

of wound closure with Uck2 overexpression at 24 hours was observed in HCC cells compared with control cells (Figure 3D1). In the transwell assay, the number of cells that passed through the Matrigel in the Bel-7402/Uck2 group was higher than that in the control groups (Figure S1A1). These results indicated that Uck2 upregulation significantly improved the migration and invasion ability of HCC cells with low metastatic potential. The endogenous Uck2 level in HCC cells with high metastatic potential was then transiently knocked down by transfecting cells with a siRNA to further confirm the effect of Uck2 on the migration and invasion of HCC cells. Two siRNAs (siRNA-\#1 and siRNA-\#2) that were designed against different regions of Uck2 effectively inhibited Uck2 expression in Sk-hep-1 cells according to
RT-qPCR and Western blotting (Figure 3C). As expected, the downregulation of endogenous Uck2 in Sk-hep-1 cells suppressed the migration and invasion abilities of the cells compared with the control cells (Figures 3D2 and S1A2). In addition, the overexpression of Uck 2 did not significantly affect the cell cycle, apoptosis, or proliferation in HCC cells (Figure S1B-D).

\section{Uck2 promotes the metastasis of HCC cells in vivo}

Since the results of our in vitro experiment suggested that alterations of Uck 2 expression regulated the migration and invasion capabilities of HCC cells, the role of the Uck2 gene in tumor metastasis in vivo was further explored. Bel- 
7402 cells with stable Uck2 expression and control cells were inoculated into the tail veins of nude mice $(n=10$, per group) for the observation of lung metastasis. The final results showed that the incidence of lung metastasis in the Bel-7402-Uck2 group was significantly increased compared with that in the control group (50\% vs 20\%) (Figure 4A). The histological analysis further confirmed that the number of lung metastatic nodules was also increased in the Bel-7402-Uck2 cells compared with the number observed in the Bel-7402-control cells (Figure 4B and C). Together, these in vivo results demonstrate the critical role of Uck2 in promoting the metastasis of HCC cells.

\section{Uck2 regulated matrix metalloproteinase expression and activation of the Stat3 signaling pathway}

As proteolytic enzymes that degrade substrates in the extracellular matrix, matrix metalloproteinases (MMPs) have long been associated with cancer cell invasion and metastasis. ${ }^{18}$ As members of the MMP family, MMP2, MMP7, and MMP9

A

\begin{tabular}{cc} 
Incidence of lung metastasis in transplanted nude mice \\
\hline & Lung metastasis \\
\hline Bel-7402-Ctrl & $2 / 10$ \\
Bel-7402-Uck2 & $5 / 10$ \\
\hline
\end{tabular}

B

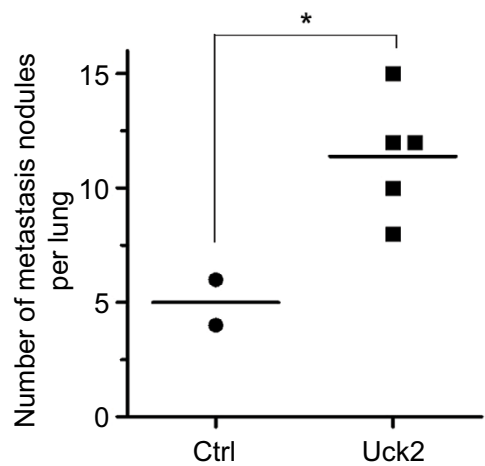

C

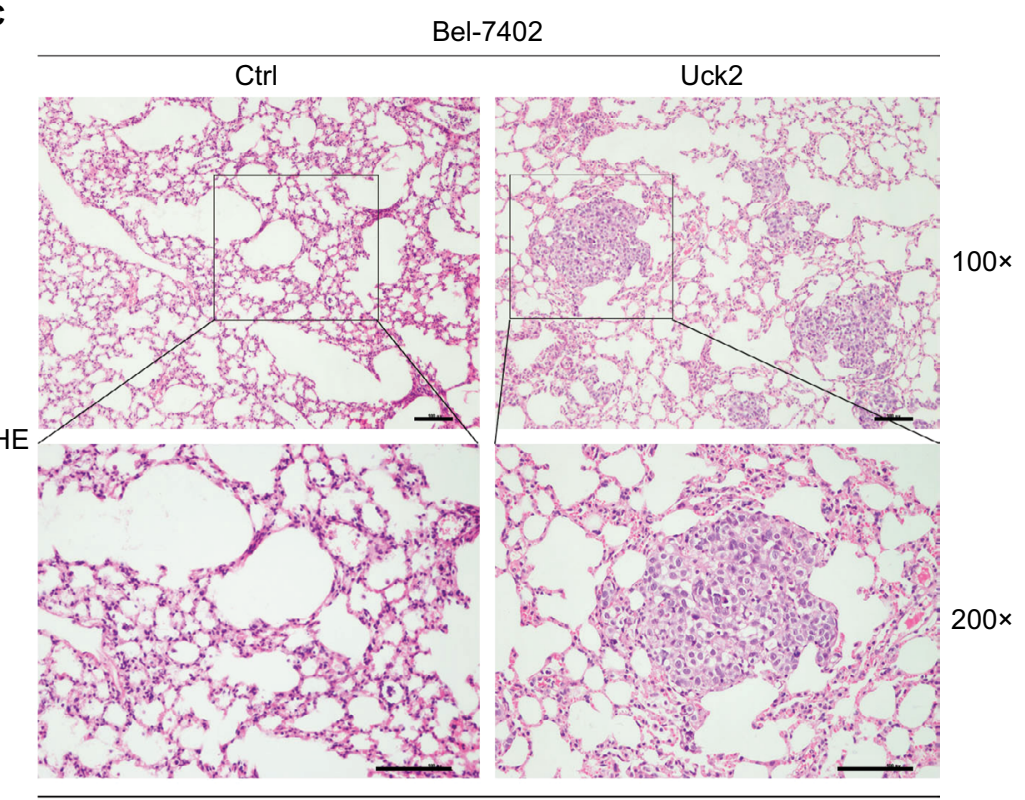

Figure 4 Uck2 promotes metastasis in HCC cells in vivo.

Notes: (A) Incidence of lung metastasis in transplanted nude mice 8 weeks after the injection of Bel-7402-Uck2 cells or control cells into the tail vein. (B) The number of metastatic lung foci observed in each group. *P-value $<0.05$. (C) Representative $H \& E$ staining images of lung tissue samples from the different experimental groups (scale bar, $100 \mu \mathrm{m})$.

Abbreviations: HCC, hepatocellular carcinoma; Uck2, uridine-cytidine kinase 2. 
have been widely studied, and we detected the expression of these proteins in Bel-7402/Uck2 and Bel-7402/control cells. The results showed that the upregulation of the Uck2 gene obviously increased the expression of MMP2 and MMP9 but had only a slight influence on the expression of MMP7 (Figure 5A). As the epithelial to mesenchymal transition (EMT) has also been accepted as a potential mechanism underlying cancer metastasis, ${ }^{19}$ we then explored the role
A

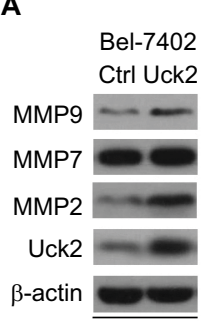

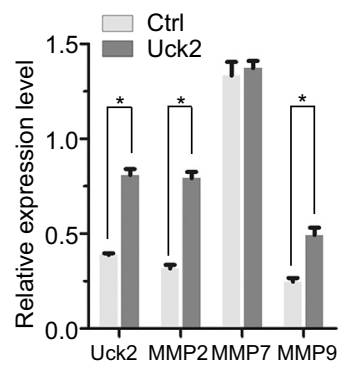

B

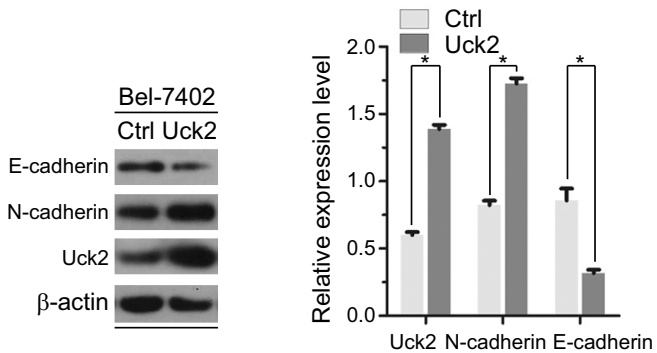

C
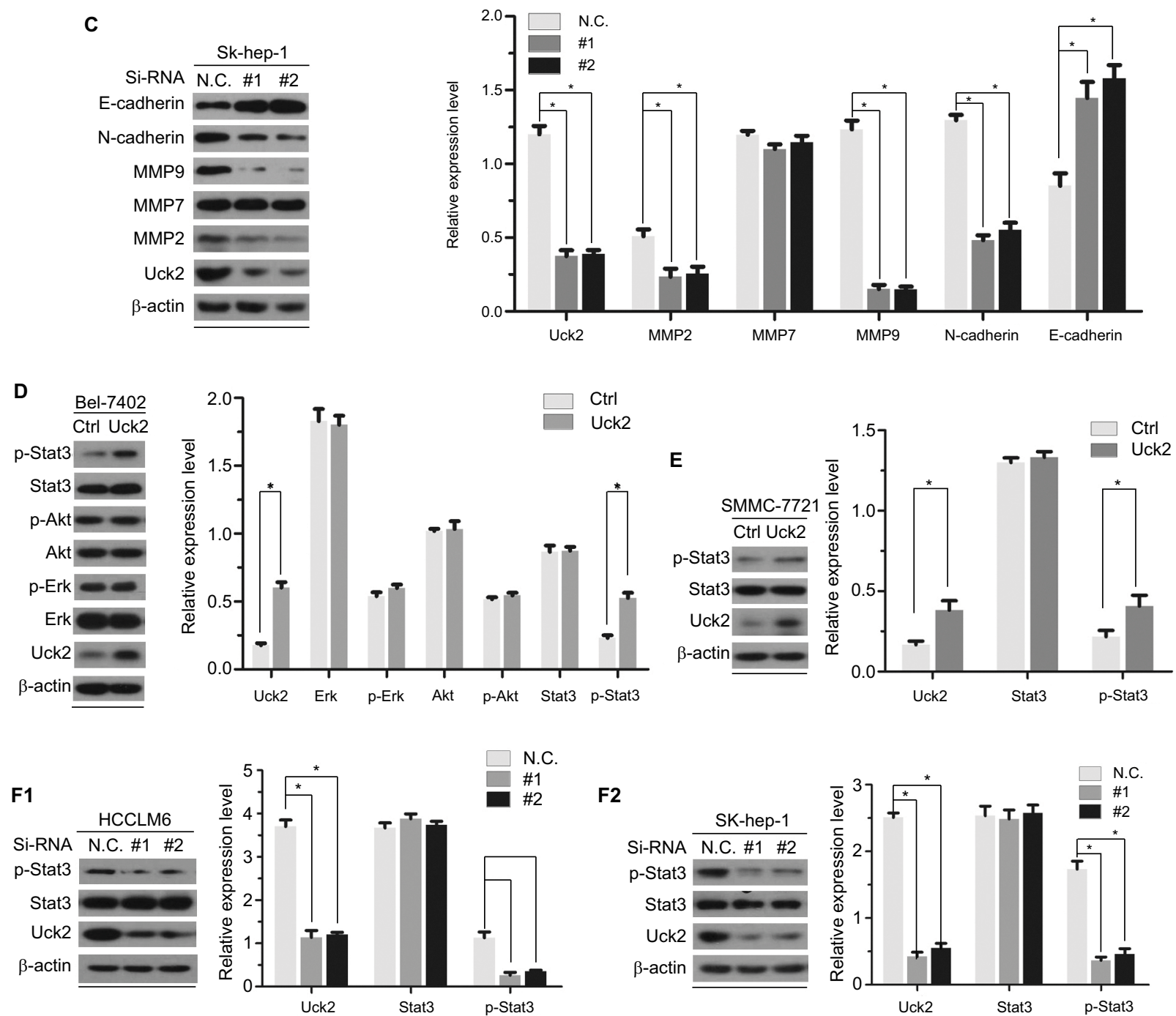

F2
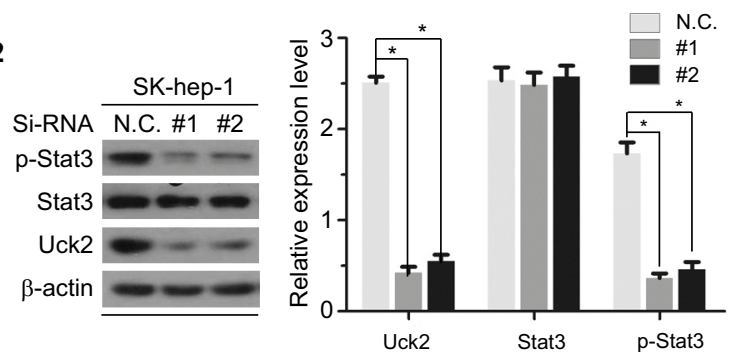

Figure 5 Uck2 regulated MMPs expression and Stat3 activation according to Western blotting analysis.

Notes: The results of Western blotting analysis are shown by raw photos and densitometry analysis. $\beta$-actin was used as the housekeeping gene to normalize expression levels in the densitometry analysis by Imagej software (I.48 version; NIH, Bethesda, MD, USA). (A) Raw photos and densitometry analysis: stable expression of Uck2 in Bel-7402 cells increased the protein levels of MMP2 and MMP9. (B) Raw photos and densitometry analysis: stable expression of Uck2 in Bel-7402 cells increased the protein level of N-cadherin and decreased the protein level of E-cadherin. (C) Raw photos and densitometry analysis: transient downregulation of Uck2 by siRNA in Sk-hep-I cells decreased the protein levels of MMP2, MMP9, and N-cadherin and increased the protein level of E-cadherin. (D) Raw photos and densitometry analysis: exploration of the oncogenic pathways regulated by the Uck2 gene. Stable expression of Uck2 in Bel-7402 cells increased the protein level of p-Stat3. (E) Raw photos and densitometry analysis: the stable expression of Uck2 in SMMC-772I cells increased the protein level of p-Stat3. (FI and F2) Raw photos and densitometry analysis: transient downregulation of Uck2 by siRNA in HCCLM6 cells and Sk-hep-I cells decreased the protein level of p-Stat3. The experiments were repeated three times, and the results are shown as the mean $\pm S D ; * P<0.05$, which indicates a significant difference by Student's $t$-test.

Abbreviations: Ctrl, control; MMP, matrix metalloproteinase; Uck2, uridine-cytidine kinase 2. 
of the Uck2 gene in regulating EMT in HCC cells. Western blot analysis showed that the upregulation of the Uck2 gene in Bel-7402 cells resulted in decreased expression of E-cadherin and increased expression of N-cadherin, but it had no effect on the expression of vimentin and fibronectin (Figure 5B). Because functional loss of E-cadherin has long been considered as the hallmark of EMT, ${ }^{20}$ the Uck2 gene might regulate the EMT in HCC cells. We further confirmed the effect of the Uck2 gene on the expression of MMP2, MMP7, MMP9, E-cadherin, and N-cadherin in Uck2 knockdown cells. The results showed that the downregulation of Uck2 in Sk-hep-1 cells significantly reduced MMP2, MMP9, and $\mathrm{N}$-cadherin expression (Figure 5C). In addition, inhibiting Uck2 expression in Sk-hep-1 cells also increased the expression of E-cadherin, but had no influence on the expression of MMP7 (Figure 5C). Furthermore, we scrutinized the potential signaling pathways activated by the Uck2 gene and found that stable overexpression of the Uck2 gene in Bel-7402 cells significantly promoted the phosphorylation of Stat3, but had little effect on the phosphorylation of Akt and Erk (Figure 5D). Overexpressing the Uck2 gene in SMMC-7721 cells also caused a significant upregulation of the phosphorylation level of Stat3 (Figure 5E). In contrast, the phosphorylation of Stat3 was inhibited after knocking down endogenous Uck2 expression in both HCCLM6 cells and Sk-hep-1 cells (Figure 5F1 and F2).

\section{Uck2 regulated the expression of MMPs and EMT progression via the Stat3 signaling pathway}

Several studies have suggested that the Stat3 signaling pathway can promote tumor invasion and metastasis by increasing the expression of MMP2 and MMP9. ${ }^{21-23}$ And this pathway was also thought to regulate EMT progression. ${ }^{24-26}$ Therefore, we next explored whether Uck2 regulated the expression of MMPs and EMT progression via the Stat3 signaling pathway. Western blot analysis showed that the upregulation of Uck2 in Bel-7402 cells increased the expression of MMP2, MMP9, and N-cadherin and decreased the expression of E-cadherin, as observed previously (Figure 6A). After treatment with $3 \mu \mathrm{M}$ WP1066 (a Stat3 inhibitor) for 24 hours, the expression levels of MMP2, MMP9, and N-cadherin were decreased and the expression of E-cadherin was increased, while Uck2 expression was unchanged (Figure 6A). These results indicated that the Uck2 gene regulated the expression of MMP2/9 and EMT progression via the Stat3 signaling pathway. Furthermore, we examined the migration and invasion capabilities of Bel-7402/Uck2 cells after treatment with a Stat3 inhibitor ( $3 \mu \mathrm{M}$ WP1066) for 24 hours. As shown in Figure $6 \mathrm{~B}$ and $\mathrm{C}$, the number of migrating and invading cells from WP1066-treated cultures decreased, demonstrating that Uck 2 facilitated the migration and invasion of HCC cells via the Stat3 signaling pathway.

\section{Discussion}

$\mathrm{HCC}$ is a common malignant tumor worldwide and has a poor survival rate. ${ }^{1,3}$ Current treatments for HCC patients are not satisfactory, and novel therapeutic targets are urgently required. Uck 2 catalyzes the phosphorylation of uridine and cytidine and their respective synthetic analogues. ${ }^{6}$ Previous studies have primarily focused on the ability of Uck 2 to activate antitumor prodrugs and to regulate chemosensitivity in cancer cells. ${ }^{8,9}$ Recently, some studies have begun to evaluate the potential prognostic value of Uck2. Shen et al reported that Uck2 expression was elevated in breast cancer tissue and associated with poor overall survival. ${ }^{10}$ In this study, we also found that Uck2 expression was significantly upregulated in HCC tissues when compared with their adjacent nontumorous tissues (Figures 1 and 2). A high Uck2 expression level was significantly correlated with tumor encapsulation, microvascular invasion, and distant metastasis (Table 1). In addition, patients with high Uck2 expression in HCC tissues had early recurrence and a poor prognosis (Figure 2D and E). Thus, Uck2 expression might be a crucial prognostic indicator of HCC.

Since previous studies have not widely considered the effects of Uck2 on the malignant biological behavior of cancer cells, we further explored the role of the Uck2 gene in HCC cells. Using wound-healing and transwell assays, we showed that the expression of Uck2 promoted the migration and invasion of HCC cells in vitro (Figure 2). We further demonstrated that the overexpression of Uck2 promoted the metastasis of Bel-7402 cells in vivo (Figure 3 ). These results are consistent with the above conclusion that a high Uck2 expression level is significantly correlated with tumor encapsulation, microvascular invasion, and distant metastasis.

A leading cause of cancer-related death is the dissemination of metastatic tumor cells and the outgrowth of secondary tumors at distant sites. MMPs and EMT play important roles in $\mathrm{HCC}$ invasiveness and metastasis. ${ }^{27}$ Invasion and metastasis into peripheral tissues require the crossing of several physical barriers, such as the endothelial basement membrane. The central role of MMPs in cancer is the degradation and remodeling of the endothelial basement membrane, paving the way through the peripheral tissue for 

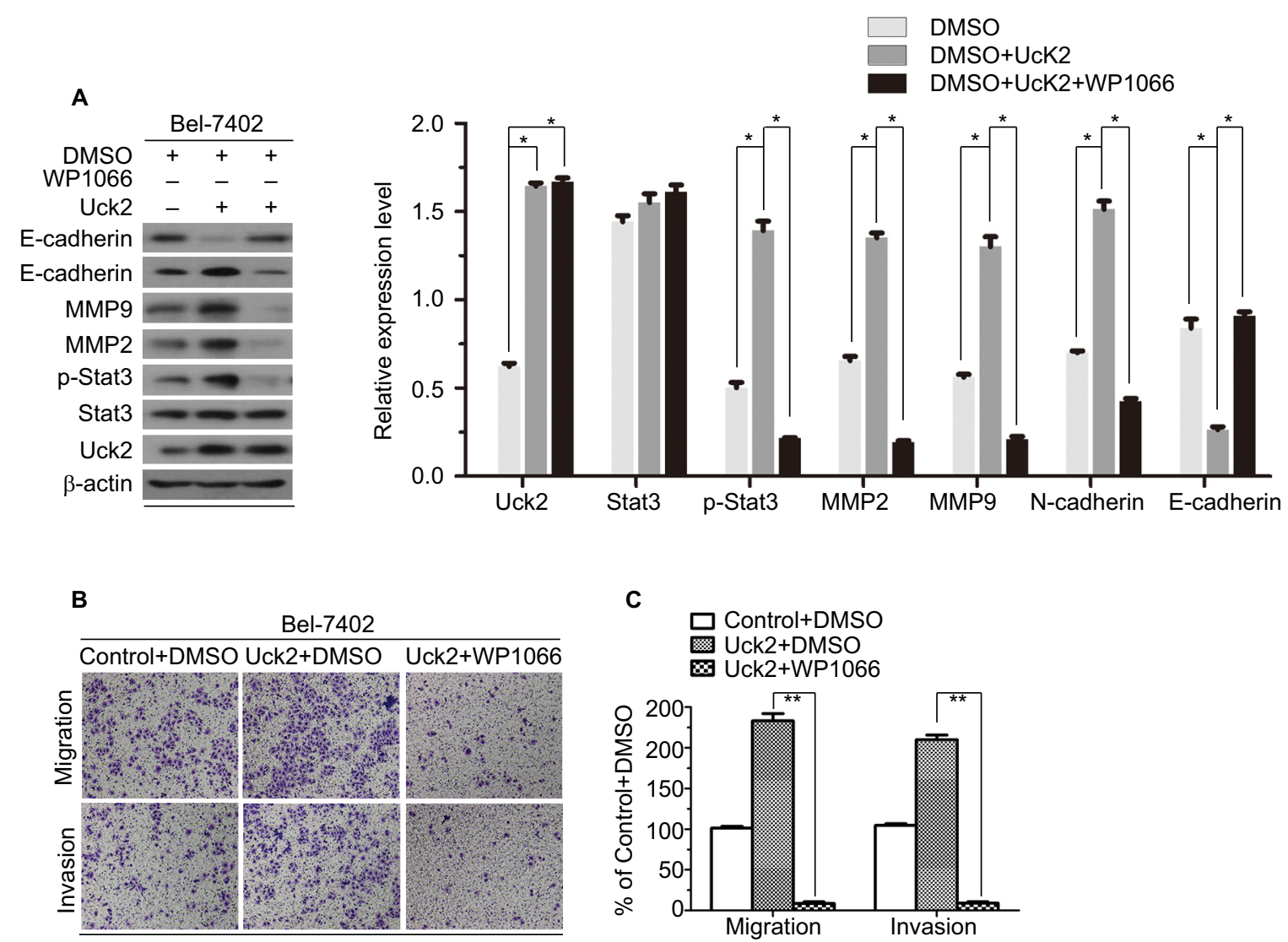

Figure 6 Uck2 regulated the expression of MMPs and EMT progression via the Stat3 signaling pathway.

Notes: (A) Raw photos and densitometry analysis of Western blotting analysis: treatment with the Stat3 inhibitor WPI066 neutralized the upregulation of MMP2 and MMP9 and reversed the EMT progression caused by the overexpression of Uck2 in Bel-7402 cells. (B and C) After treatment with a Stat3 inhibitor (3 $\mu$ M WPI066) for 24 hours, Bel-7402/Uck2 cells showed decreased migration ability and decreased invasion ability by a transwell assay. $\beta$-actin was used as the housekeeping gene to normalize expression levels in the densitometry analysis by ImageJ software (I.48 version; NIH, Bethesda, MD, USA). The experiments were repeated three times, and the results are shown as the mean $\pm S D ; * P<0.05$, $* * P<0.01$, which indicates a significant difference by Student's $t$-test.

Abbreviations: EMT, epithelial to mesenchymal transition; MMP, matrix metalloproteinase; Uck2, uridine-cytidine kinase 2.

invasion and metastasis. ${ }^{28}$ We found that the upregulation of Uck2 increased the expression of MMP2 and MMP9, which might contribute to the role of Uck2 in promoting the invasion abilities of HCC cells (Figure 5A). In addition, the overexpression of Uck2 resulted in decreased expression of E-cadherin (an epithelial marker) and increased expression of $\mathrm{N}$-cadherin (a mesenchymal marker) (Figure 5B). Therefore, we proposed that Uck 2 could promote the EMT progression in HCC cells. The conversion from the epithelial state to the mesenchymal state involves profound phenotypic changes in cancer cells that include the loss of cell-cell adhesion, the loss of cell polarity, and the acquisition of migratory and invasive properties. ${ }^{29}$

Persistent activation of Stat 3 has been frequently observed in $\mathrm{HCC},{ }^{12,13}$ resulting in modulated expression of genes that promote tumor cell survival, proliferation, metastasis, and angiogenesis. ${ }^{30}$ The results of Western blotting in our study showed that Uck2 upregulated the expression of p-Stat3 (Figure 5D). Courapied et al found that Cdk5 kinase could interact with Stat 3 to induce its phosphorylation. ${ }^{31}$ This finding indicated that Uck2, as a kinase, might also associate with Stat3 to activate the Stat3 signaling pathway. Several studies have shown a relationship between Stat3 activation and MMPs expression. Xie et al demonstrated that Stat-3 activation regulates the expression of MMP2 through direct interaction with the MMP2 promoter; MMP2 then mediates tumor invasion and metastasis. ${ }^{32}$ Song et al showed that stable expression of active Stat 3 increases MMP9 expression in MCF-7 cells, which is dependent on its interactions with Fra-1/c-Jun to activate the MMP9 gene promoter. ${ }^{33}$ Xiong et al demonstrated that Stat3 mediates EMT progression of colorectal cancer through regulating the expression of E-cadherin and N-cadherin and that the Stat3-induced decrease in E-cadherin and cell invasion depended on activation of the 
EMT inducer ZEB1 in colorectal cancer cells. ${ }^{25}$ Therefore, we proposed that Uck2 might upregulate the expression of MMP2/9 and promote EMT progression via activating the Stat3 signaling pathway, which mediates tumor invasion and metastasis. We found that treatment with WP1066, a Stat3 inhibitor, neutralized the upregulation of MMP2 and MMP9 and reversed the EMT progression caused by the overexpression of Uck2 in Bel-7402 cells (Figure 6A). Furthermore, the enhanced migration and invasion capacities induced by Uck2 overexpression were obviously weakened after treatment with WP1066 (Figure 6B and C). Further studies are needed to explore the mechanism of Uck2-induced Stat3 signaling activation in regulating the expression of MMPs and EMT progression in HCC cells.

\section{Conclusion}

The current study is the first to demonstrate the important role of Uck2 in HCC. We show that Uck2 expression is elevated in HCC tissues compared with that in normal control tissues at both the mRNA and protein levels. The high expression of Uck 2 in HCC tissues is associated with early recurrence and poor prognosis in patients. The expression of Uck 2 in HCC cells regulates the cellular capacity for migration and invasion but not proliferation. Finally, we showed that Uck2 can upregulate the expression of MMP2/9 and promote cell migration and invasion via the Stat 3 signaling pathway.

\section{Ethics approval and informed consent}

The study protocol was approved by the Institute Review Board of SYSU. The patient consent was written informed consent, and this procedure was conducted in accordance with the Declaration of Helsinki. All experimental procedures involving animals were in accordance with the National Institutes of Health guide for the care and use of laboratory animals and approved by the Animal Ethical and Welfare Committee of SYSU (DD-17-0906).

\section{Data availability}

The datasets used and/or analyzed during the current study are available from the corresponding author on reasonable request.

\section{Acknowledgments}

This study was supported by the National Natural Science Funds (grant no. 81672403), the Natural Science Foundation of Guangdong, China (grant no. 2016A030311051), and the Science and Technology Program of Guangzhou, China (grant no. 201607010225).

\section{Author contributions}

All authors made substantial contributions to conception and design, acquisition of data, or analysis and interpretation of data; took part in drafting the article or revising it critically for important intellectual content; gave final approval of the version to be published; and agree to be accountable for all aspects of the work.

\section{Disclosure}

The authors report no conflicts of interest in this work.

\section{References}

1. Siegel RL, Miller KD, Jemal A. Cancer statistics, 2015. CA Cancer J Clin. 2015;65(1):5-29.

2. Chen W, Zheng R, Baade PD, et al. Cancer statistics in China, 2015. CA Cancer J Clin. 2016;66(2):115-132.

3. Goh GB, Chang PE, Tan CK. Changing epidemiology of hepatocellular carcinoma in Asia. Best Pract Res Clin Gastroenterol. 2015;29(6):919-928.

4. Lane AN, Fan TW. Regulation of mammalian nucleotide metabolism and biosynthesis. Nucleic Acids Res. 2015;43(4):2466-2485.

5. Tomoike F, Nakagawa N, Fukui K, et al. Indispensable residue for uridine binding in the uridine-cytidine kinase family. Biochem Biophys Rep. 2017;11:93-98.

6. van Rompay AR, Norda A, Lindén K, Johansson M, Karlsson A. Phosphorylation of uridine and cytidine nucleoside analogs by two human uridine-cytidine kinases. Mol Pharmacol. 2001;59(5):1181-1186.

7. Muhale FA, Wetmore BA, Thomas RS, Mcleod HL. Systems pharmacology assessment of the 5-fluorouracil pathway. Pharmacogenomics. 2011;12(3):341-350.

8. Murata D, Endo Y, Obata T, et al. A crucial role of uridine/cytidine kinase 2 in antitumor activity of 3'-ethynyl nucleosides. Drug Metab Dispos. 2004;32(10):1178-1182.

9. Sato A, Takano T, Hiramoto A, et al. Role of the uridine/cytidine kinase 2 mutation in cellular sensitiveness toward 3'-ethynylcytidine treatment of human cancer cells. Anticancer Drugs. 2017;28(7):781-786.

10. Shen G, He P, Mao Y, et al. Overexpression of Uridine-Cytidine Kinase 2 Correlates with Breast Cancer Progression and Poor Prognosis. $J$ Breast Cancer. 2017;20(2):132-141.

11. Aggarwal BB, Kunnumakkara AB, Harikumar KB, et al. Signal transducer and activator of transcription-3, inflammation, and cancer: how intimate is the relationship? Ann NY Acad Sci. 2009;1171:59-76.

12. Rajendran P, Ong TH, Chen L, et al. Suppression of signal transducer and activator of transcription 3 activation by butein inhibits growth of human hepatocellular carcinoma in vivo. Clin Cancer Res. 2011;17(6):1425-1439.

13. Subramaniam A, Shanmugam MK, Ong TH, et al. Emodin inhibits growth and induces apoptosis in an orthotopic hepatocellular carcinoma model by blocking activation of STAT3. Br J Pharmacol. 2013;170(4):807-821.

14. Wang SW, Sun YM. The IL-6/JAK/STAT3 pathway: potential therapeutic strategies in treating colorectal cancer (Review). Int J Oncol. 2014;44(4):1032-1040.

15. Rhodes DR, Yu J, Shanker K, et al. ONCOMINE: a cancer microarray database and integrated data-mining platform. Neoplasia. 2004;6(1):1-6. 
16. Chen X, Cheung ST, So S, et al. Gene expression patterns in human liver cancers. Mol Biol Cell. 2002;13(6):1929-1939.

17. Roessler S, Jia HL, Budhu A, et al. A unique metastasis gene signature enables prediction of tumor relapse in early-stage hepatocellular carcinoma patients. Cancer Res. 2010;70(24):10202-10212.

18. Egeblad M, Werb Z. New functions for the matrix metalloproteinases in cancer progression. Nat Rev Cancer. 2002;2(3):161-174.

19. Tania M, Khan MA, Fu J. Epithelial to mesenchymal transition inducing transcription factors and metastatic cancer. Tumour Biol. 2014;35(8):7335-7342.

20. Zeisberg M, Neilson EG. Biomarkers for epithelial-mesenchymal transitions. J Clin Invest. 2009;119(6):1429-1437.

21. Groner B, Lucks P, Borghouts C. The function of Stat3 in tumor cells and their microenvironment. Semin Cell Dev Biol. 2008;19(4):341-350.

22. Wei Z, Jiang X, Qiao H, et al. STAT3 interacts with Skp2/p27/p21 pathway to regulate the motility and invasion of gastric cancer cells. Cell Signal. 2013;25(4):931-938.

23. Banerjee K, Resat H. Constitutive activation of STAT3 in breast cancer cells: A review. Int J Cancer. 2016;138(11):2570-2578.

24. Wendt MK, Balanis N, Carlin CR, et al. STAT3 and epithelial-mesenchymal transitions in carcinomas. JAKSTAT. 2014;3(1):e28975.

25. Xiong $\mathrm{H}$, Hong $\mathrm{J}$, du W, et al. Roles of STAT3 and ZEB1 proteins in E-cadherin down-regulation and human colorectal cancer epithelialmesenchymal transition. J Biol Chem. 2012;287(8):5819-5832.
26. Chen J, Lan T, Zhang W, et al. Feed-Forward Reciprocal Activation of PAFR and STAT3 Regulates Epithelial-Mesenchymal Transition in Non-Small Cell Lung Cancer. Cancer Res. 2015;75(19):4198-4210.

27. Yuan M, Guo H, Li J, et al. Slit2 and Robo1 induce opposing effects on metastasis of hepatocellular carcinoma Sk-hep-1 cells. Int J Oncol. 2016;49(1):305-315.

28. Kessenbrock K, Plaks V, Werb Z. Matrix metalloproteinases: regulators of the tumor microenvironment. Cell. 2010;141(1):52-67.

29. Thiery JP, Acloque H, Huang RY, Nieto MA. Epithelial-mesenchymal transitions in development and disease. Cell. 2009;139(5):871-890.

30. Subramaniam A, Shanmugam MK, Perumal E, et al. Potential role of signal transducer and activator of transcription (STAT) 3 signaling pathway in inflammation, survival, proliferation and invasion of hepatocellular carcinoma. Biochim Biophys Acta. 2013;1835(1):46-60.

31. Courapied S, Sellier H, de Carné Trécesson S, et al. The cdk5 kinase regulates the STAT3 transcription factor to prevent DNA damage upon topoisomerase I inhibition. J Biol Chem. 2010;285(35):26765-26778.

32. Xie TX, Wei D, Liu M, et al. Stat3 activation regulates the expression of matrix metalloproteinase- 2 and tumor invasion and metastasis. Oncogene. 2004;23(20):3550-3560.

33. Song Y, Qian L, Song S, et al. Fra-1 and Stat3 synergistically regulate activation of human MMP-9 gene. Mol Immunol. 2008;45(1): 137-143. 


\section{Supplementary material}

A1

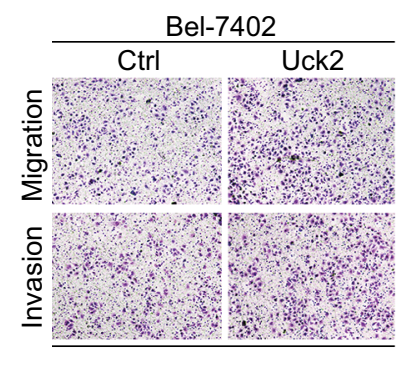

A2

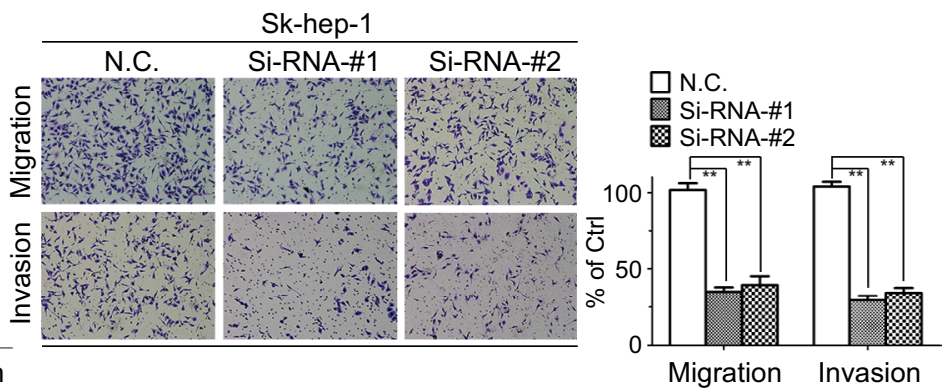

B

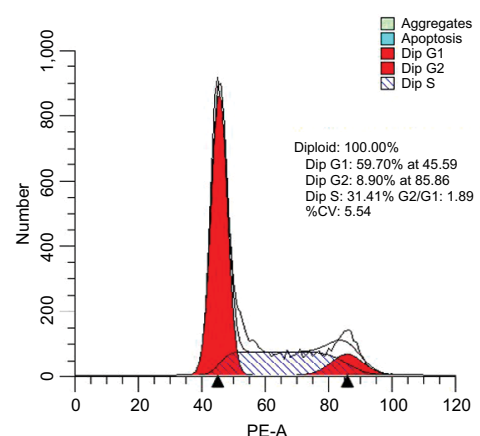

C

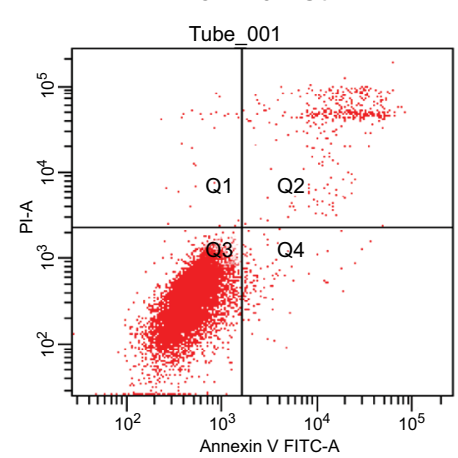

D

Bel-7402-Ctrl

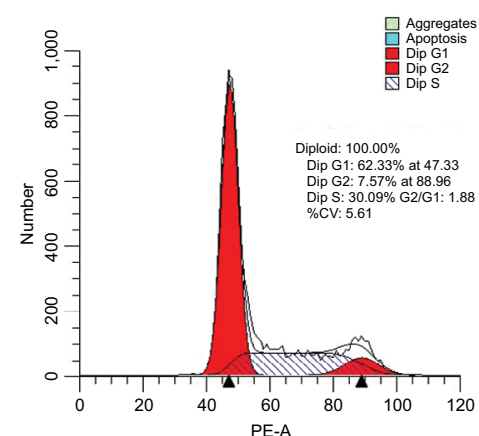

Bel-7402-Uck2

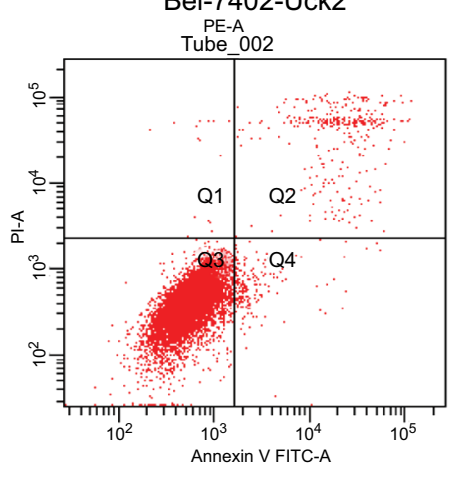

Bel-7402-Uck2

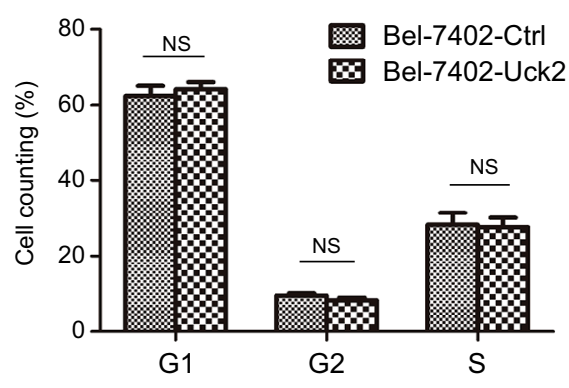

Bel-7402

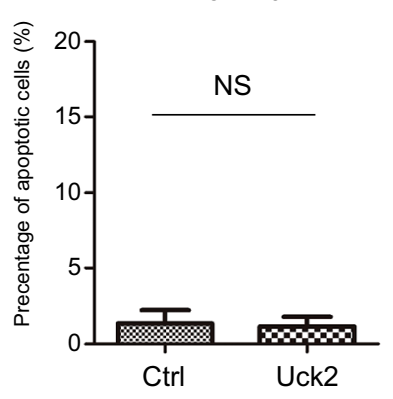

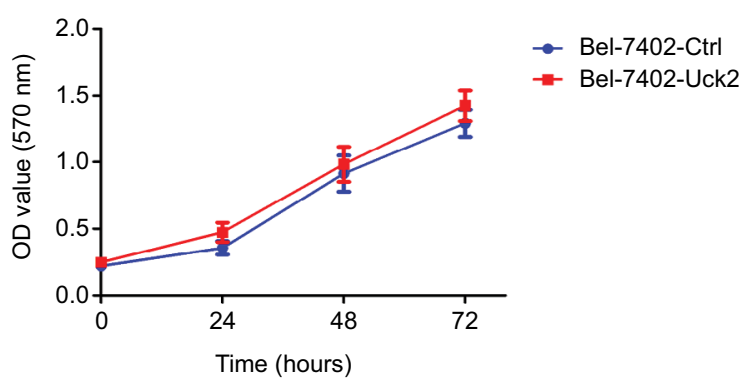

Figure SI Uck2 promoted HCC cell migration and invasion, but did not significantly affect the cell cycle, apoptosis, or proliferation.

Notes: (AI and A2) Effects of Uck2 overexpression and knockdown on HCC cell migration and invasion by transwell assays. (B) Analysis of the cell cycle by flow cytometry in Bel-7402-Ctrl and Bel-7402-Uck2 groups. (C) Analysis of apoptosis by flow cytometry in Bel-7402-Ctrl and Bel-7402-Uck2 groups. (D) MTT proliferation assay for Bel7402-Ctrl and Bel-7402-Uck2 groups. The experiments were repeated three times, and the results are shown as the mean \pm SD; NS represents not significant; $* * P<0.01$, which indicates a significant difference by an independent Student's $t$-test.

Abbreviations: Ctrl, control; CV, coefficient of variation; HCC, hepatocellular carcinoma; N.C., negative control; Si, Si-RNA; Uck2, uridine-cytidine kinase 2. 
Cancer Management and Research

\section{Publish your work in this journal}

Cancer Management and Research is an international, peer-reviewed open access journal focusing on cancer research and the optimal use of preventative and integrated treatment interventions to achieve improved outcomes, enhanced survival and quality of life for the cancer patient.

The manuscript management system is completely online and includes

Submit your manuscript here: https://www.dovepress.com/cancer-management-and-research-journal

a very quick and fair peer-review system, which is all easy to use. Visit $\mathrm{http}: / / \mathrm{www}$.dovepress.com/testimonials.php to read real quotes from published authors. 